\title{
Alterations in gut microbiota of abdominal aortic aneurysm mice
}

\author{
Jiahe Xie ${ }^{1,2,3 \dagger}$, Weiling Lu ${ }^{1,3+}$, Lintao Zhong ${ }^{4}$, Yuhua Hu ${ }^{1,3}$, Qingrui $\mathrm{Li}^{1,3}$, Rongming Ding ${ }^{1,3}$, Zhonggao Zhong ${ }^{1,3}$, \\ Ziyou Liu ${ }^{3}$, Hai Xiao ${ }^{3}$, Dongming Xie ${ }^{1,3}$, Guofu Zheng ${ }^{5}$, Bo Ye ${ }^{5}$, Yiming Zhong ${ }^{1,3^{*}}$ and Zuheng Liu ${ }^{2,6^{*}}$ (D)
}

\begin{abstract}
Background: The gut microbiome plays an important role in various cardiovascular diseases, such as atherosclerosis and hypertension, which are associated with abdominal aortic aneurysms (AAAs).

Methods: Here, we used 165 rRNA sequencing to explore gut microbiota in C57BL ApoE ${ }^{-/-}$mice with AAAs. A mouse model of abdominal aortic aneurysms was induced with angiotensin II (Ang II) (1000 ng/min per kg). On day 28 after the operation, fecal samples were collected and stored at $-80^{\circ} \mathrm{C}$ until DNA extraction. We determined the relative abundances of bacterial taxonomic groups using 165 rRNA amplicon metabarcoding, and sequences were analyzed using a combination of mother software and UPARSE.

Results: We found that the gut microbiome was different between control and AAA mice. The results of correlation analysis between AAA diameter and the gut microbiome as well as LEfSe of the genera Akkermansia, Odoribacter, Helicobacter and Ruminococcus might be important in the progression of AAAs.

Conclusions: AAA mice is subjected to gut microbial dysbiosis, and gut microbiota might be a potential target for further investigation.
\end{abstract}

Keywords: Abdominal aortic aneurysm, Gut microbiota, Akkermansia

\section{Background}

Abdominal aortic aneurysm (AAA), a vascular disease with high disability and mortality, is associated with atherosclerosis and hypertension [1]. An abnormal vascular inflammatory response and abnormal vascular structure trigger the onset of aortic aneurysms.

Recent studies have suggested that gut microbiota probably participate in host inflammation and the formation of atherosclerosis and hypertension [2, 3]. Gut microbiota have been shown to be closely related to systemic inflammation by generating some toxic metabolic factors or by releasing lipopolysaccharides [4]. Any factors that influence the intestinal microenvironment likely interrupt the balance in gut microbiota, ultimately leading to changes in the gut microbiome. For example, high choline intake

\footnotetext{
* Correspondence: yimingdoc@126.com; john_lau@126.com

${ }^{\dagger}$ Jiahe Xie and Weiling Lu contributed equally to this work.

'Department of Cardiology, First Affiliated Hospital of Gannan Medical University, Ganzhou, China

${ }^{2}$ State Key Laboratory of Organ Failure Research, Department of Cardiology,

Nanfang Hospital, Southern Medical University, Guangzhou, China

Full list of author information is available at the end of the article
}

elevates the level of blood trimethylamine oxide (TMAO), a metabolic factor that promotes cordial hypertrophy and ultimately increases the incidence of various cardiovascular- and cerebrovascular-related diseases [5-9], while even changes in diet can change the gut miceobiome [10-12]. All these factors can change the gut microbiome.

Therefore, focusing on the gut microbiome might provide some possible mechanism of AAAs. In this study, we applied Ang II-induced AAA to $\mathrm{ApoE}^{-/-}$mice and investigated alterations in the gut microbiome.

\section{Methods}

Abdominal aortic aneurysm mouse model

Five- to six-month-old male C57BL ApoE ${ }^{-/-}$mice were obtained from the laboratory of the animal center of Gannan Medical University and were used in this study. All mice were housed in cages and bred in a temperaturecontrolled room maintained at $22-26^{\circ} \mathrm{C}$ on a 12 -h lightdark cycle with standard food and water. This study was approved by the Gannan Medical University animal ethics committee, and the use of animals in this study was in

(c) The Author(s). 2020 Open Access This article is distributed under the terms of the Creative Commons Attribution 4.0 International License (http://creativecommons.org/licenses/by/4.0/), which permits unrestricted use, distribution, and 
A

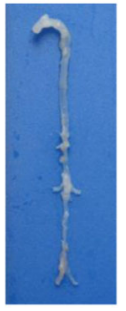

Control

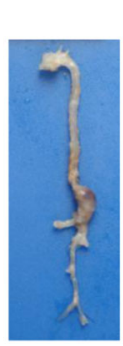

AAA
B

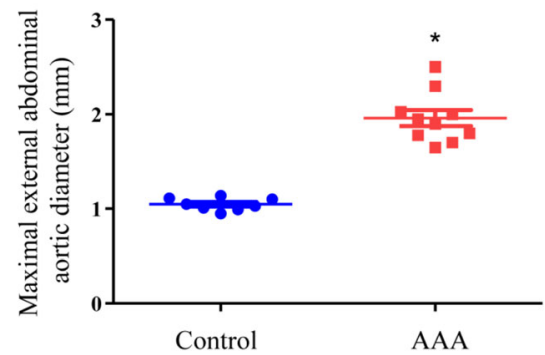

Fig. 1 a Representative images of abdominal aortic aneurysms. b A scatter plot of maximal external abdominal aortic diameter. ${ }^{*} P<0.05, n=8-10$

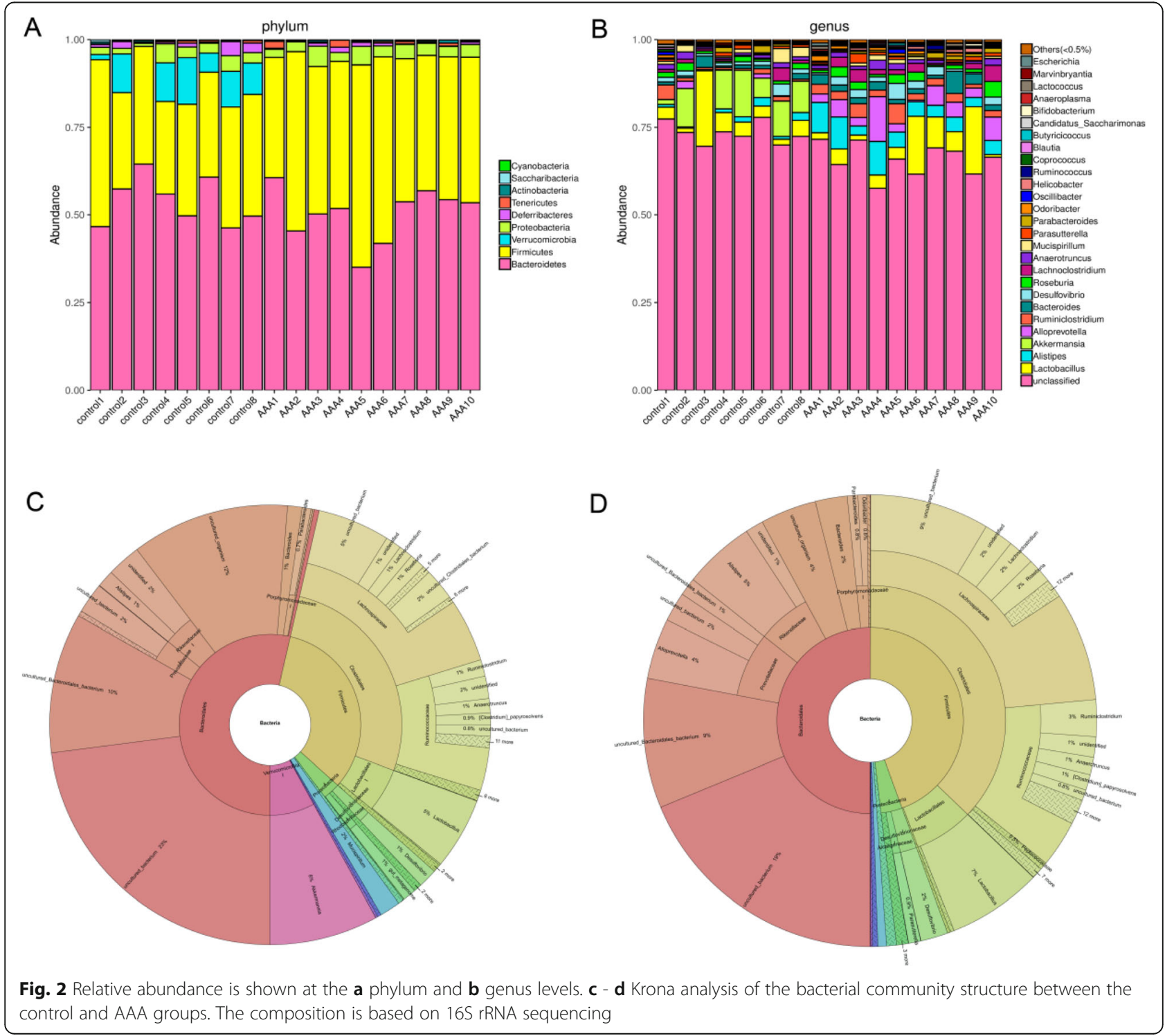


compliance with the Guide for the Care and Use of Laboratory Animals (NIH, 8th Edition, 2011). The mice used in this study were anesthetized with a mixture of xylazine $(5 \mathrm{mg} / \mathrm{kg})$ and ketamine $(100 \mathrm{mg} / \mathrm{kg})$ by intraperitoneal injection. A mouse model of abdominal aortic aneurysm or sham was induced with angiotensin II $(1000 \mathrm{ng} / \mathrm{min}$ per $\mathrm{kg}, n=17)$ or saline $(n=8)$ by Alzet Osmotic Pump implantation, which has been described previously [13]. After 28 days, fecal samples were collected and stored at $-80^{\circ} \mathrm{C}$ until DNA extraction. After fecal samples collection, mice were sacrificed by overdose of pentobarbital $(500 \mathrm{mg} / \mathrm{kg})$, then the abdominal aorta were separated. Among them, four mice died in observation period which probably caused by the rupture of arterial aneurysm, while another 3 mice were excluded because the increased vessel diameter were less than $50 \%$.

\section{DNA extraction}

Samples were stored at $-80^{\circ} \mathrm{C}$ until DNA extraction. The DNA was extracted from $200 \mathrm{mg}$ samples using the QIAamp DNA Stool Mini Kit (QIAGEN, Hilden, Germany) following the manufacturer's instructions. The DNA concentration and purity were checked by running the samples on $1.0 \%$ agarose gels.

\section{S rRNA amplification and sequencing}

The methods were described previously [14]. Briefly, $16 \mathrm{~S}$ rRNA genes were amplified by using V3-V4 regions bacterial primers (357F 5' - ACTCCTACGGRAGGCAG CAG-3' and 806R5'- GGACTACHVGGGTWTCTAAT $\left.-3^{\prime}\right)$. The primers also contained the Illumina $5^{\prime}$ overhang adapter sequences. The libraries were sequenced on the MiSeq PE300 sequencing platform using a MiSeq v3 Reagent Kit (Illumina).

\section{Bioinformatic analysis}

Bioinformatic analysis were described in previous study [15]. Briefly, after the raw data were demultiplexed based on the barcode, low-quality base pairs were removed by using parameters with SLIDINGWINDOW: 50:20 MINLEN: 50 . Then, $16 \mathrm{~S}$ rRNA sequences were analyzed by using mothur software (version 1.33.3), UPARSE (usearch version v8.1.1756,), and R (version 3.2.3). For the alpha-diversity analysis, Shannon, Chaol, ACE and PD_whole_ tree were calculated by using mother, while the beta-diversity metrics, the weighted and unweighted UniFrac distance were calculated by using mothur and visualized with principal coordinate analysis ( $\mathrm{PCoA})$ and tree by $\mathrm{R}$.

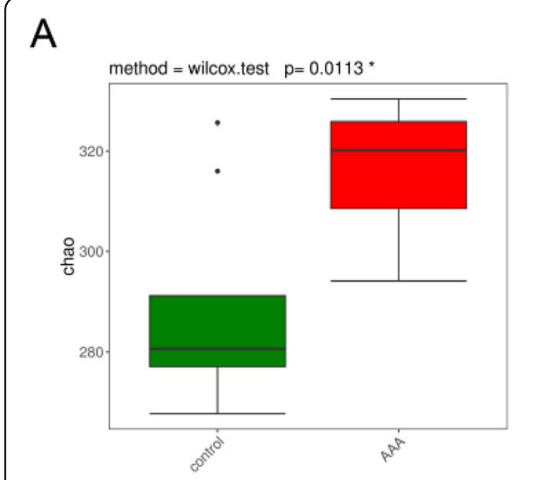

B

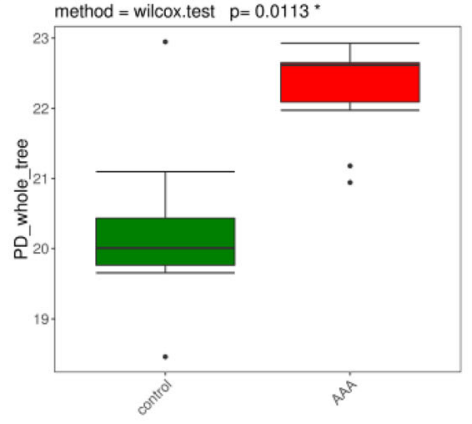

C

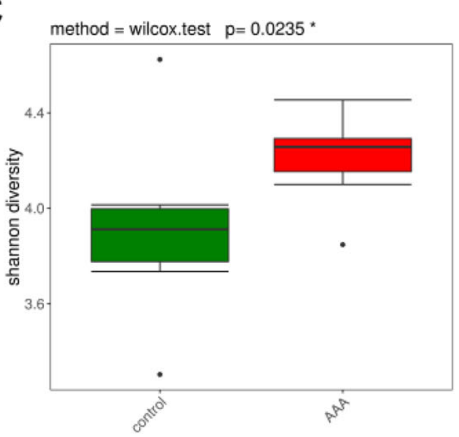

D

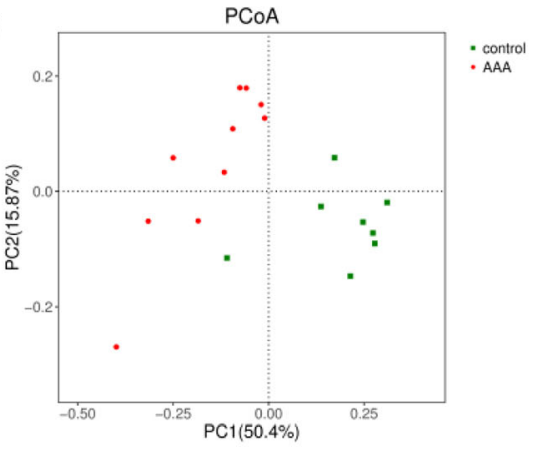

Weighted_unifrac distance
E

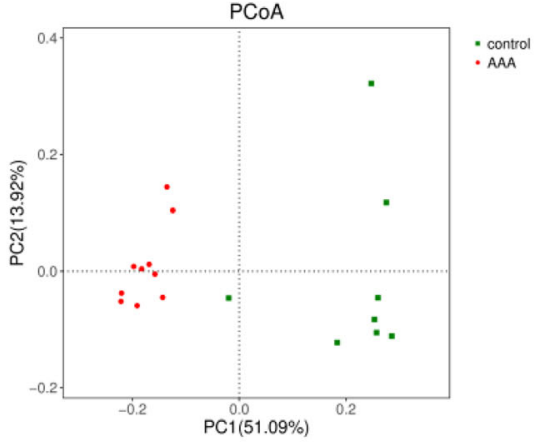

Unweighted_unifrac distance

Fig. 3 Alpha diversity of the a Chao index, b PD_whole tree and c Shannon index between the control and AAA groups. Principal coordinate analysis (PCOA) of gut microbiota for $\mathbf{d}$ Weighted_unifrac distance and e Unweighted_unifrac distance from AAA mice and control mice. (Red dots, AAA group; Green dots, control group) 


\section{Statistical analysis}

Quantitative data were displayed as mean \pm SEM (standard error of mean). Comparison between two experimental groups was based on a two-tailed t-test. In all analyses, differences were considered statistically significant at a value of $P<0.05$.

\section{Results}

The abdominal aortic aneurysm model was induced by angiotensin II

After an overdose of anesthesia, the mice were euthanized. Then, the abdominal aortas were dissected, and the AAAs were visualized, as shown in Fig. 1a. The maximal external abdominal aortic diameter was measured using ImageJ software, which showed a significant difference between the AAA and control groups $(n=8-10, P<0.05)$.
The gut microbial composition changed in the abdominal aortic aneurysm mouse model

To explore the gut microbiome in AAA mice, fecal samples were collected before the mice were sacrificed. After bioinformatic analysis, we found that Bacteroidetes, Firmicutes, Verrucomicrobia and Proteobacteria in the taxonomic composition plots at the phylum level represented most of the bacterial community of the gut (Fig. 2a). At the genus level, we found that Akkermansia was reduced in AAA mice (Fig. 2b), and this result was also demonstrated by Krona analysis (Fig. 2c-d).

The alpha and beta diversities of the gut microbiome between the AAA and control groups

Then, principal coordinate analysis (PCoA) of gut microbiota from AAA mice and control mice were performed.

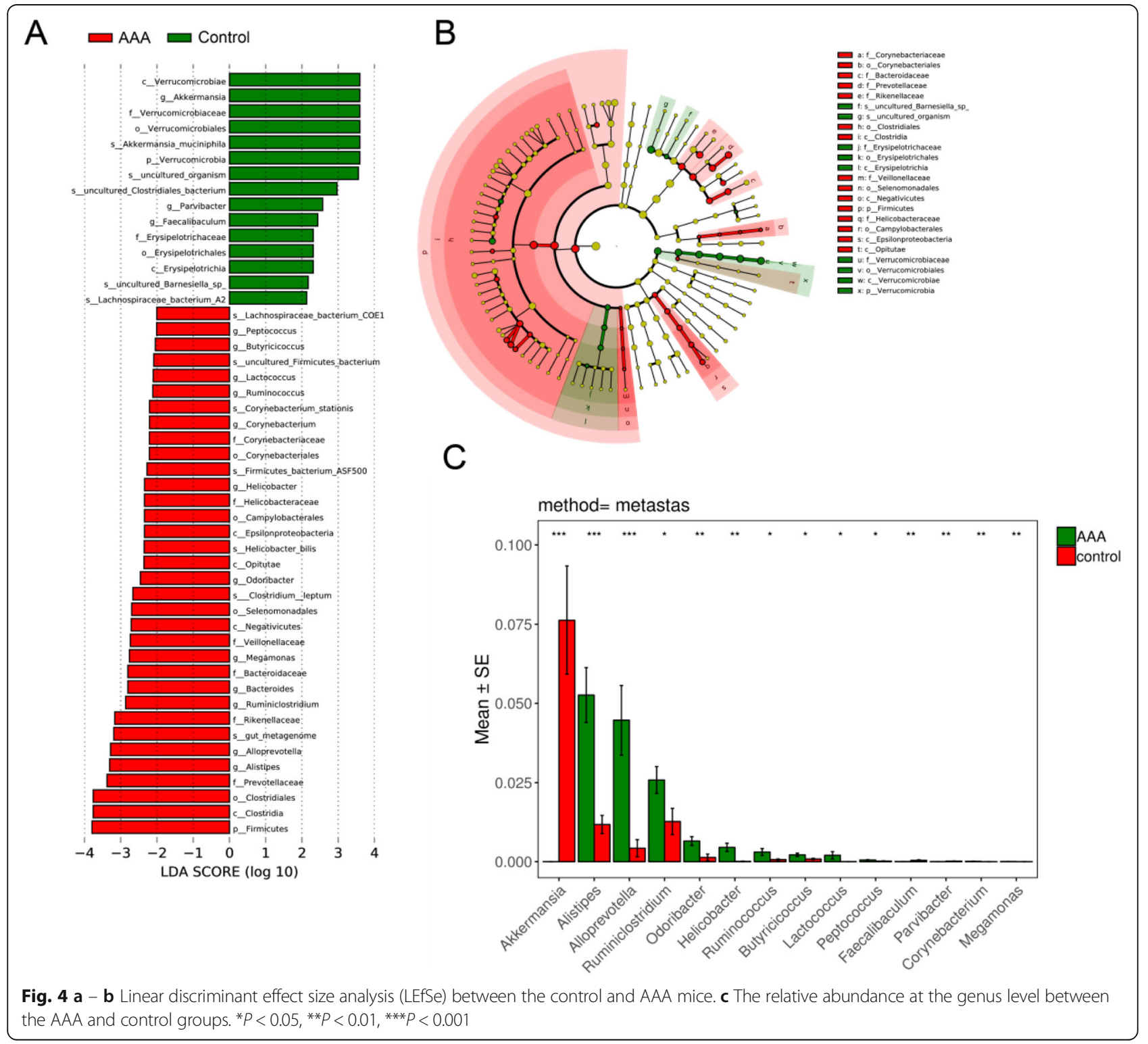


We found that AAA mice had higher (Fig. 3a) Chao index $(P<0.05)$, (Fig. 3b) PD_whole tree $(\mathrm{P}<0.05)$ and (Fig. 3c) Shannon index $(P<0.05)$ levels than control mice. In addition, beta diversity was shown by (Fig. 3d) Weighted_unifrac distance $(P<0.05)$ and (Fig. 3e) Unweihted_unifrac distance $(P<0.05)$. Together, these results indicate that the gut microbiome might participate in the progression of AAA.

The LDA effect size analysis (LEfSe) revealed the differences between the AAA and control groups

To identify distinctive features between these groups, LEfSe was performed (Fig. 4a-b). The results of an LDA effect size analysis showed that species from AAkkermansia. muciniphila and Lachnospiraceae bacterium A2 were significantly higher in the control group than in the AAA group, while six species were increased in AAA mice: Lachnospiraceae bacterium, COE1, Corynebacterium stationis, Firmicutes Bacterium ASF500, Helicobacter bilis and Clostridium leptum (Fig. 4a-b). Differential analysis by metastas also revealed a similar result (Fig. 4c); thus, Akkermansia, Alistipes, Alloprevotella, Ruminiclostridium, Odoribacter and Helicobacter might be important in AAAs.

The correlation between the abdominal aortic diameter and the gut microbiome

To explore the correlation between the severity of AAA and the gut microbiome, Spearman correlation was used. The $\mathrm{R}$ value, $P$ value and FDR are shown in Fig. 5a, while the heat map is shown in Fig. 5b. We found that 2 genera (Akkermansia and Parvibacter) were negatively correlated with the diameter of AAA, whereas 7 genera (Odoribacter, Helicobacter, Ruminococcus, Megamonas, Bacteroides, Alistipes, and Alloprevotella) were significantly positively correlated with the diameter. These findings suggest that Akkermansia and Parvibacter might be essential in the treatment of AAAs.

\section{Discussion}

In the present study, we found that abdominal aortic aneurysm (AAA) mice were subject to gut microbial dysbiosis. Although gut microbiome alterations emerged after the induction of AAAs, gut microbial dysbiosis likely inversely promoted the course of disease. Thus, exploring the gut microbiome in AAAs might provide some clues in the progress of AAAs.

First, we found that the gut microbial community was different between the AAA group and the corresponding control group. This result might be due to the challenge of angiotensin II, an endogenous, rigorous vasoconstrictor that can promote cardiac hypertrophy and impair vascular endothelial cells [16]. The stimulation of Ang II might lead to vasoconstriction in the intestine and thus further interrupt the gut microbiome [17]. Similar results were found in high blood pressure patients, as we found that the genus Alistipes, harbored in HBP patients [17], was positively correlated with the diameter of an AAA. However, our findings in the gut microbiome were not completely consistent with previous findings in a hypertension mouse model induced by Ang II [17]. These differences might be due to the knock-out of the $A p o E$ gene, as gut microbiota interact with host genes [18]. Another explanation is the

\begin{tabular}{l|rrr} 
A & & & \\
\multicolumn{1}{|c}{ Genus } & \multicolumn{2}{r}{ R value P value } & FDR \\
Akkermansia & -0.738 & $<0.001$ & 0.011 \\
Odoribacter & 0.514 & 0.029 & 0.155 \\
Helicobacter & 0.662 & 0.003 & 0.027 \\
Ruminococcus & 0.490 & 0.039 & 0.187 \\
Parvibacter & -0.626 & 0.005 & 0.042 \\
Megamonas & 0.715 & 0.001 & 0.014 \\
Bacteroides & 0.478 & 0.045 & 0.196 \\
Alistipes & 0.684 & 0.002 & 0.021 \\
Alloprevotella & 0.620 & 0.006 & 0.042
\end{tabular}

B

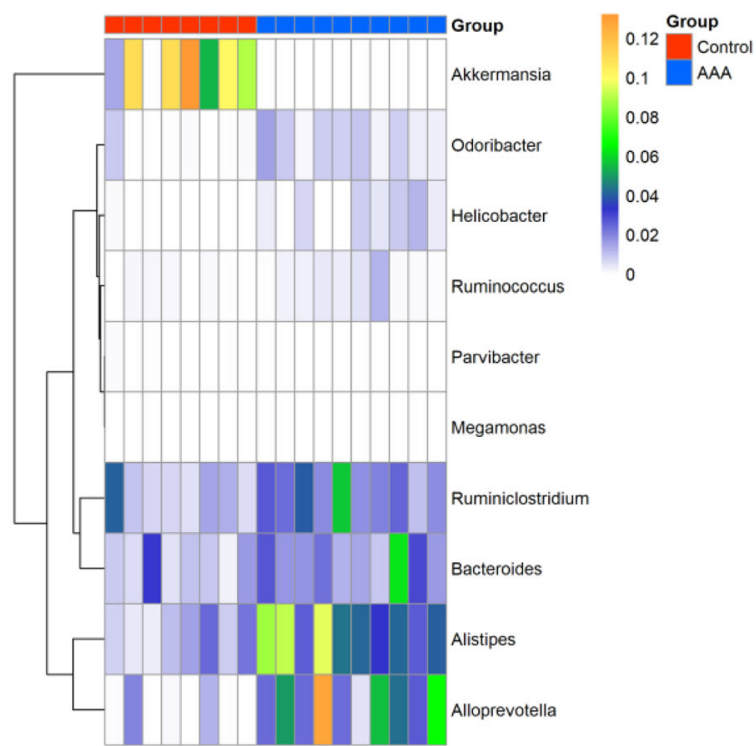

Fig. 5 a Correlations between the gut microbiome at the genus level and the maximal external abdominal aortic diameter. $\mathbf{b}$ A heatmap of the gut microbiome between the control and AAA groups 
different symbiotic bacteria in these studies, as different regions also influence the gut microbiome [19].

We also found that $A$. muciniphila, a newly identified probiotic reported in recent years [20], was significantly reduced in the AAA mice. It is not surprising that $A$. muciniphila might be involved in the progression of AAAs because it exerts beneficial effects in various diseases including atherosclerosis [21] and dyslipidemia [22]. Other studies also found that Akkermansia decreased with increasing age in Tibetan minipigs [23], and it was found to be induced by some types of traditional Chinese medicine [24].

A. muciniphila is a kind of mucin-producing bacteria that can repair damage to the intestinal barrier in atherosclerosis models of $\mathrm{ApoE}^{-/-}$mice [21]. In addition, we previously found that exercise training could increase the abundance of Akkermansia [20]. These results indicate that $A$. muciniphila is likely essential in the host body and that it might be essential in the progress of AAAs.

In addition, a species belonging to Helicobacter was also increased in AAA mice. This result was similar to that of our previous study in myocardial infarction mice, as Helicobacter was negatively associated with left ventricular ejection fraction in MI mice [20], indicating that Helicobacter might be a common pathogen underlying some cardiovascular diseases.

The AAA model was established with a sustained infusion of angiotension II. Although previous studies have shown that the gut microbiome changes after challenge with angiotensin II or dyslipidemia, they have not investigated both of these effects. Additionally, AAA patients in the clinic always exhibit dyslipidemia and abnormal blood pressure. Nevertheless, further studies are still required to verify the function of these diseases.

There are still some limitations should be concerned. First, the mechanism of AAA seems more complicated in clinical practice rather than the abnormal of reninangiotensin system or simply the deficiency of $A p o E$ gene. Second, the reduction of $A$. muciniphila in AAA mice is an observational result, more data form human analysis is needed, and the underlying mechanisms of $A$. muciniphila should be investigated in the future.

\section{Conclusions}

AAA mice is accompanied with gut microbial dysbiosis.

\section{Abbreviations \\ AAAs: Abdominal aortic aneurysms; Ang II: Angiotensin II; LEfSe: LDA effect size analysis; PCOA: The principal coordinate analysis; TMAO: Trimethylamine} oxide

\section{Acknowledgements}

Not applicable.

\section{Authors' contributions}

Substantial contributions to the conception or design of the study or the acquisition, analysis, or interpretation of data used in the study: $X J H, L W L$, ZLT, HYH, LQR, DRM, ZZG, LZY, XH, ZYM, XDM, ZGF, YB and LZH. XJH, LWL and $\mathrm{LZH}$. Drafting the study or revising it critically for important intellectual content: LZH, YB, XJH and LWL. Final approval of the version to be published: $\mathrm{LZH}, \mathrm{YB}, \mathrm{XJH}, \mathrm{XDM}$ and LWL. Agreement to be accountable for all aspects of the study and ensuring that questions related to the accuracy or integrity of any part of the study are appropriately investigated and resolved: $X J H, L W L, Z L T, H Y H, L Q R, D R M, Z Z G, L Z Y, X H, Z Y M, X D M, Z G F, Y B$ and $L Z H$.

All authors read and approved the final manuscript.

\section{Funding}

This study was supported by "China Postdoctoral Science Foundation funded project (No. 2019M652959)" (Supporting the design of the study, collection, analysis, interpretation of data) (Supporting 165 rRNA sequencing), the "Science and Technology Research Program of Department of Education of Jiangxi Province in China (No. GJJ170850, GJJ180813 and GJJ170876)" (Supporting the manuscript writing, collection and interpretation of data) (Purchasing Alzet Osmotic Pumps), the "Open Project of Key Laboratory of Prevention and Treatment of Cardiovascular and Cerebrovascular Diseases, Ministry of Education (No.XN201813)" (Supporting the collection of data) (Purchasing Angiotensin II and the drugs in anesthesia), and the Scientific Research Project of Gannan Medical University (No.TD201704)" (Supporting the collection of data) (Purchasing and raising the mice used in this study).

\section{Availability of data and materials}

All relevant data is presented in the manuscript and the datasets used and analyzed during the current study are available from the corresponding author on reasonable request. The sequences were deposited in the

European Nucleotide Archive (ENA) under the accession number PRJEB34405

\section{Ethics approval and consent to participate}

This study was approved by the Gannan Medical University review board, and the use of animals in this study was in compliance with the Guide for the Care and Use of Laboratory Animals (NIH, 8th Edition, 2011).

\section{Consent for publication}

Not applicable.

\section{Competing interests}

The authors declare that they have no competing interests.

\section{Author details}

${ }^{1}$ Department of Cardiology, First Affiliated Hospital of Gannan Medical University, Ganzhou, China. ${ }^{2}$ State Key Laboratory of Organ Failure Research, Department of Cardiology, Nanfang Hospital, Southern Medical University, Guangzhou, China. ${ }^{3}$ Key Laboratory of Prevention and Treatment of Cardiovascular and Cerebrovascular Diseases, Ministry of Education, Gannan Medical University, Ganzhou, People's Republic of China. ${ }^{4}$ Department of Cardiology, Zhuhai Hospital, Jinan University, Zhuhai, China. ${ }^{5}$ Department of Vascular Surgery, the Affiliated Ganzhou Hospital of Nanchang University, Guangzhou, China. ${ }^{6}$ Key Laboratory for Organ Failure Research, Ministry of Education of the People's Republic of China, Southern Medical University, Guangzhou, China.

Received: 23 August 2019 Accepted: 8 January 2020

Published online: 28 January 2020

\section{References}

1. Li W, Luo S, Luo J, Liu Y, Ning B, Huang W, et al. Predictors associated with increased prevalence of abdominal aortic aneurysm in Chinese patients with atherosclerotic risk factors. Eur J Vasc Endovasc Surg. 2017;54(1):43-9.

2. Liu Z, Li J, Liu H, Tang Y, Zhan Q, Lai W, et al. The intestinal microbiota associated with cardiac valve calcification differs from that of coronary artery disease. Atherosclerosis. 2019;284:121-8.

3. Sun Y, Zhong L, He X, Wang S, Lai Y, Wu W, et al. LncRNA H19 promotes vascular inflammation and abdominal aortic aneurysm formation by functioning as a competing endogenous RNA. J Mol Cell Cardiol. 2019;131: 66-81. 
4. Gong S, Yan Z, Liu Z, Niu M, Fang H, Li N, et al. Intestinal microbiota mediates the susceptibility to Polymicrobial sepsis-induced liver injury by Granisetron generation in mice. Hepatology. 2019;69(4):1751-67.

5. Li Z, Wu Z, Yan J, Liu H, Liu Q, Deng Y, et al. Gut microbe-derived metabolite trimethylamine $\mathrm{N}$-oxide induces cardiac hypertrophy and fibrosis. Lab Investig. 2019:99(3):346-57.

6. Yin J, Liao SX, He Y, Wang S, Xia GH, Liu FT, et al. Dysbiosis of Gut Microbiota With Reduced Trimethylamine-N-Oxide Level in Patients With Large-Artery Atherosclerotic Stroke or Transient Ischemic Attack. J Am Heart Assoc. 2015;4(11). https://doi.org/10.1161/JAHA.115.002699, https://www. ahajournals.org/doi/full/10.1161/JAHA.115.002699.

7. Nie J, Xie L, Zhao BX, Li Y, Qiu B, Zhu F, et al. Serum Trimethylamine Noxide concentration is positively associated with first Stroke in hypertensive patients. Stroke. 2018;49(9):2021-8.

8. Xu KY, Xia GH, Lu JQ, Chen MX, Zhen X, Wang S, et al. Impaired renal function and dysbiosis of gut microbiota contribute to increased trimethylamine-N-oxide in chronic kidney disease patients. Sci Rep. 2017; 7(1):1445.

9. Lin A, Zheng W, He Y, Tang W, Wei $X$, He R, et al. Gut microbiota in patients with Parkinson's disease in southern China. Parkinsonism Relat Disord. 2018; 53:82-8.

10. Hu J, Luo H, Wang J, Tang W, Lu J, Wu S, et al. Enteric dysbiosis-linked gut barrier disruption triggers early renal injury induced by chronic high salt feeding in mice. Exp Mol Med. 2017;49(8):e370.

11. Liu F, Li P, Chen M, Luo Y, Prabhakar M, Zheng H, et al. Fructooligosaccharide (FOS) and Galactooligosaccharide (GOS) increase Bifidobacterium but reduce butyrate producing Bacteria with adverse glycemic metabolism in healthy young population. Sci Rep. 2017;7(1):11789.

12. Wu G, Niu M, Tang W, Hu J, Wei G, He Z, et al. L-Fucose ameliorates highfat diet-induced obesity and hepatic steatosis in mice. J Transl Med. 2018; 16(1):344

13. Zhong L, He X, Si X, Wang H, Li B, Hu Y, et al. SM22alpha (smooth muscle 22alpha) prevents aortic aneurysm formation by inhibiting smooth muscle cell phenotypic switching through suppressing reactive oxygen species/NFkappaB (nuclear factor-kappaB). Arterioscler Thromb Vasc Biol. 2019;39(1): e10-25

14. Zhao J, Yao Y, Li D, Xu H, Wu J, Wen A, et al. Characterization of the gut microbiota in six geographical populations of Chinese rhesus macaques (Macaca mulatta), implying an adaptation to high-altitude environment. Microb Ecol. 2018;76(2):565-77.

15. Feng W, Wang H, Zhang P, Gao C, Tao J, Ge Z, et al. Modulation of gut microbiota contributes to curcumin-mediated attenuation of hepatic steatosis in rats. Biochim Biophys Acta Gen Subj. 2017;1861(7):1801-12.

16. Liu Z, Hua J, Cai W, Zhan Q, Lai W, Zeng Q, et al. Nterminal truncated peroxisome proliferatoractivated receptorgamma coactivatorlalpha alleviates phenylephrineinduced mitochondrial dysfunction and decreases lipid droplet accumulation in neonatal rat cardiomyocytes. Mol Med Rep. 2018;18(2):2142-52.

17. Kim S, Goel R, Kumar A, Qi Y, Lobaton G, Hosaka K, et al. Imbalance of gut microbiome and intestinal epithelial barrier dysfunction in patients with high blood pressure. Clin Sci. 2018;132(6):701-18.

18. Li Y, Hao Y, Fan F, Zhang B. The Role of Microbiome in Insomnia, Circadian Disturbance and Depression. Front Psychiatry. 2018:9:669.

19. He Y, Wu W, Zheng HM, Li P, McDonald D, Sheng HF, et al. Regional variation limits applications of healthy gut microbiome reference ranges and disease models. Nat Med. 2018;24(10):1532-5.

20. Liu Z, Liu HY, Zhou H, Zhan Q, Lai W, Zeng Q, et al. Moderate-intensity exercise affects gut microbiome composition and influences cardiac function in myocardial infarction mice. Front Microbiol. 2017:8:1687.

21. Li J, Lin S, Vanhoutte PM, Woo CW, Xu A. Akkermansia Muciniphila protects against atherosclerosis by preventing metabolic Endotoxemia-induced inflammation in Apoe-/- mice. Circulation. 2016;133(24):2434-46.

22. Shen J, Tong $X$, Sud N, Khound R, Song Y, Maldonado-Gomez MX, et al. Low-density lipoprotein receptor signaling mediates the triglyceridelowering action of Akkermansia muciniphila in genetic-induced hyperlipidemia. Arterioscler Thromb Vasc Biol. 2016;36(7):1448-56.

23. Jiang X, Chen B, Gu D, Rong Z, Su X, Yue M, et al. Gut microbia compositions in four age groups of Tibetan Minipigs. Pol J Microbiol. 2018; 67(3):383-8.
24. Liu CS, Liang X, Wei XH, Jin Z, Chen FL, Tang QF, et al. Gegen Qinlian decoction treats diarrhea in piglets by modulating gut microbiota and short-chain fatty acids. Front Microbiol. 2019;10:825.

\section{Publisher's Note}

Springer Nature remains neutral with regard to jurisdictional claims in published maps and institutional affiliations.
Ready to submit your research? Choose BMC and benefit from:

- fast, convenient online submission

- thorough peer review by experienced researchers in your field

- rapid publication on acceptance

- support for research data, including large and complex data types

- gold Open Access which fosters wider collaboration and increased citations

- maximum visibility for your research: over $100 \mathrm{M}$ website views per year

At $\mathrm{BMC}$, research is always in progress.

Learn more biomedcentral.com/submissions 